\title{
Cost-Effectiveness of a Team-Based Integrative Medicine Approach to the Treatment of Back Pain
}

\author{
Peter M. Wayne, PhD, ${ }^{1,2}$ Julie E. Buring, ScD, ${ }^{1,2}$ David M. Eisenberg, MD, ${ }^{3}$ Kamila Osypiuk, MS, \\ Brian J. Gow, BS, ${ }^{1}$ Roger B. Davis, ScD, Claudia M. Witt, MD, 5,6,* and Thomas Reinhold, PD Dr ${ }^{6, *}$
}

\begin{abstract}
Objectives: To report the results of health economic analyses comparing two treatment approaches for chronic low back pain (CLBP).

Design: Observational prospective cohort study comparing effectiveness and cost-effectiveness of CLBP care provided at an integrative care clinic with that provided in other clinics within the same hospital. CLBP-related medical utilization, function, quality of life, and days of work incapacity were self-reported at baseline, 3, 6, and 12 months.

Settings/Location: Osher Clinical Center (OCC) based at a tertiary academic hospital (Brigham and Women's Hospital $[\mathrm{BWH}])$ and other clinics at BWH.

Subjects: CLBP patients seeking care at OCC or non-OCC BWH clinics.

Interventions: Integrative or conventional care for CLBP as prescribed by the treating clinician(s).

Outcome measures: Quality-adjusted life years (QALYs) were estimated per treatment approach based on the SF-12. Cost per QALY gained was evaluated using an incremental cost-effectiveness ratio (ICER). ICERs based on CLBP-specific effectiveness measures (Roland Disability Questionnaire [RDQ] and bothersomeness of pain [BOP]) were exploratory outcomes.

Results: Total adjusted annual CLBP-related costs per patient were greater in the OCC versus non-OCC group (\$11,526.73 vs. \$6,810.63). Between group differences in QALYs were small and ICER estimate of cost per QALY gained was high $(\$ 436,676)$. However, unadjusted mean direct costs per patient decreased over time in the OCC group. Savings in direct costs of $\$ 391$ (95\% confidence interval: $-1,078$ to 1,861 ) were observed in the OCC group for the 6- to 12-month period, driven primarily by reduced medication usage. ICERs based on adjusted RDQ and BOP group differences showed cost of $\$ 2,073$ and $\$ 4,203$ for a one-point reduction per respective scale.

Conclusions: When adjusted for baseline differences, self-reported costs were higher in the OCC group with only small effects on QALYs. However, trends toward decreased direct expenditures and medication usage over time warrant further investigation. Future studies evaluating potential benefits of integrative care models for the management of CLBP should employ randomized designs, longer observational periods, and explore multiple metrics of cost-effectiveness.
\end{abstract}

Keywords: chronic low back pain, integrative medicine, cost-effectiveness, interdisciplinary care

\footnotetext{
${ }^{1}$ Osher Center for Integrative Medicine, Harvard Medical School and Brigham and Women's Hospital, Boston, MA.

${ }^{2}$ Division of Preventive Medicine, Brigham and Women's Hospital, Boston, MA.

${ }^{3}$ Department of Nutrition, Harvard T.H. Chan School of Public Health, Boston, MA.

${ }^{4}$ Division of General Medicine and Primary Care, Beth Israel Deaconess Medical Center, Harvard Medical School, Boston, MA.

${ }^{5}$ Institute for Complementary and Integrative Medicine, University Hospital Zurich, University of Zurich, Zürich, Switzerland.

${ }^{6}$ Institute of Social Medicine, Epidemiology, and Health Economics, Charité-Universitätsmedizin Berlin, corporate member of Freie Universität Berlin, Humboldt-Universität zu Berlin, and the Berlin Institute of Health, Berlin, Germany.

*Both these authors are co-senior authors.
} 


\section{Introduction}

C HRONIC LOW BACK PAIN (CLBP) is a highly prevalent, burdensome, and costly health problem in Western industrialized countries. ${ }^{1-3}$ Despite considerable investment, basic and clinical research have not translated into a decreased prevalence of CLBP, nor in the development of unimodal conventional medical approaches that result in consistently and markedly improved efficacy. ${ }^{4,5}$ This has led many patients to seek complementary and integrative therapies (CIT) for CLBP. ${ }^{6,7}$ Surveys support that CLBP patients are among the highest users of CIT, $6,8,9$ and it is not uncommon for them to use more than one CIT at the same time, and to integrate these therapies with conventional care. ${ }^{9-11}$

The high prevalence of use of CIT for CLBP has catalyzed clinical research evaluating the safety and effectiveness of these modalities. ${ }^{12,13}$ However, less attention has been devoted to economic evaluation of the cost-effectiveness of CIT for back pain, and especially to models of care that integrate CIT within multimodal programs delivered in academic hospital settings. ${ }^{14}$ This is critical as cost-effectiveness of novel therapeutic strategies, along with safety and clinical effectiveness, are essential to informing patient and health care policy decisions.

In 2007, the Brigham and Women's Hospital (BWH) and Harvard Medical School launched the Osher Clinical Center (OCC) for Complementary and Integrative Medical Therapies. The clinic is situated within the ambulatory center of the hospital and can be utilized by any BWH patient without referral. OCC clinical services include chiropractic, acupuncture, psychiatry, physician-administered integrative medicine consultations, and multiple mind-body and movement-based therapies. A unique feature of the clinic is its extensive training of an integrated multidisciplinary team of clinicians to enhance interprofessional communication and understanding, and to optimize shared care. ${ }^{15,16}$ A primary focus of the clinic is the treatment of musculoskeletal pain.

We conducted an observational comparative effectiveness study comparing CLBP patients who received CIT at the OCC to CLBP patients receiving usual care at other clinics in the same hospital. ${ }^{17}$ We found that, at 12 months, CLBP patients who received $\mathrm{CIT}$ at the OCC exhibited significant and clinically meaningful benefits in functional status and significant, but clinically small benefits in bothersomeness of pain (BOP). ${ }^{17}$

In the present article, we report the results of health economic analyses comparing these two treatment approaches for CLBP. First, we compare CLBP-related medical utilization and expenditures reported over 12 months from a societal perspective (including direct costs paid by patients and/or reimbursed by health insurance and indirect costs due to work incapacity). Second, we estimate quality-adjusted life years (QALYs) for each treatment approach based on SF-12 utility data $^{18}$ and analyze the cost per QALY gained using an incremental cost-effectiveness ratio (ICER) approach. Finally, in additional exploratory cost-effectiveness analyses, we estimate ICERs by using CLBP-specific effectiveness measures, including metrics of back pain-related function and BOP.

\section{Materials and Methods}

\section{Study design}

Details of the background, design, and methods for the parent study have been previously published. ${ }^{17}$ In brief, this observational prospective cohort study was designed to compare the effectiveness and cost-effectiveness of CLBP care provided by the OCC team compared with CLBP care provided in other settings in the same hospital (non-OCC). All CLBP patients at BWH could choose to receive care at the OCC, either by their personal choice or as a referral from a $\mathrm{BWH}$ provider. Both the OCC and the non-OCC patients were treated as per usual care of the treating clinicians. No treatments were provided by the investigators themselves. Patients were observed over a 12-month period.

Patients were included if they had a diagnosis of nonspecific CLBP or had herniated nucleus pulposus or stenosis. CLBP was defined, as in prior studies, as 3 months or more of CLBP or 6 months or more of intermittent low back pain. ${ }^{19}$ Participants with CLBP related to cancer, fracture, or infection were excluded. Participants were $\geq 21$ years of age and English speaking and had to agree to three follow-up assessments by phone over a period of 12 months. All participants provided written informed consent. The study was approved by Partners HealthCare Institutional Review Board and was registered at ClinicalTrials.gov (Identifier NCT01355237).

\section{Medical utilization and cost data}

Utilization of medical resources related to CLBP for all participants was systematically collected with structured self-report instruments at baseline, and at the 3-, 6-, and 12month follow-up interview. Participants documented use of the therapeutic resources for CLBP over the prior 3 months (at baseline and the 3- and 6-month interviews) and the prior 6 months (for the 12-month interview) in the following eight categories: daily activities (e.g., exercise classes, nonmedical therapies), devices (e.g., support pillows), emergency room (ER) visits, hospital stays, injections, surgeries, medications, and office visits (to both conventional and CIT providers). Additionally, data on days of work incapacity and reduced working hours related to CLBP were collected.

Total costs per patient were estimated by multiplying reported resource consumption by estimates of mean costs as follows: ER visits were based on Blue Cross Blue Shield of Massachusetts (BCBSMA) cost estimates ${ }^{20}$; hospital stays were based on the 2012 Agency for Healthcare Research and Quality estimate of average cost per stay ${ }^{21}$; injections and surgeries were based on American Medical Association CPT/Medicare payment searches with MD input $^{22}$; office visits were based on BCBSMA ${ }^{20}$ (conventional providers) and expert input and web-derived average Bostonarea costs (CIT providers); medications were based on costs listed on RED BOOK Online ${ }^{\circledR 23}$ (prescription) and Drugstore.com (over-the-counter, OTC); exercise classes were based on web-derived average Boston-area costs; and devices were based on costs listed on Amazon.com.

For estimation of indirect costs, we assumed a value of $\$ 30$ per hour absent from work as used in other studies. ${ }^{24}$ The total cost at a given time period was taken as the sum of costs in each of the nine categories. Total annual costs were calculated by summarizing the costs over all time periods. For patients with data availability of less than the 12-month study duration, the existing cost data were extrapolated to an annual timeframe.

\section{Utility and clinical outcomes}

Our primary parameter for assessing cost-effectiveness was QALYs based on the SF-12 assessments at baseline, 
and 3, 6, and 12-month follow-ups. Therefore, the SF-12 quality-of-life (QOL) data were converted into SF-6D using an algorithm developed by Brazier and Roberts. ${ }^{25}$ For the SF-6D, preference weights are available, on which the derivation of health state utilities in our analysis was based. Minimum requirements for QALY calculation were: (1) existing SF-12 data at baseline; and (2) at least one existing SF-12 follow-up dataset. For patients who did not fulfill these minimum requirements QALYs were not calculated. Full year QALYs were calculated using the area under the curve method, which assumes linear changes between longitudinal utility values. ${ }^{26}$

Two secondary outcomes were also used for additional exploratory cost-effectiveness analyses. Back pain-related functional limitation was assessed with the modified Roland Disability Questionnaire (RDQ). ${ }^{27}$ Higher scores (range 023 ) indicate greater functional limitation. BOP in the past week was measured on a $0-10$ scale ( 0 indicating "not at all bothersome"; 10 indicating "extremely bothersome"). ${ }^{28}$ For both RDQ and BOP, we defined patients with a clinically meaningful $\geq 30 \%$ improvement as responders. ${ }^{28}$

\section{Cost-effectiveness}

Comparisons of cost-effectiveness between the OCC and non-OCC groups were assessed using an ICER approach, ${ }^{29}$ including all patients with existing data on annual costs and QALYs. ICERs calculate the difference in costs between two interventions divided by the difference in effects and are typically employed if both effects and costs are found to be greater in an intervention group versus control group. The ICER was calculated as additional U.S. dollars per one effectiveness unit gained (here: QALY [primary], RDQ point, RDQ responder [ $\geq 30 \%$ improvement], BOP point, and BOP responder):

$$
\begin{array}{r}
\text { ICER }=\left(\text { Total } \text { cost }_{\text {OCC }}-\text { Total }_{\text {cost }} \text { non }- \text { OCC }\right) / \\
\left(\text { Effects }_{\text {OCC }}-\text { Effects }_{\text {non }}-\text { OCC }\right)
\end{array}
$$

For the primary outcome (additional costs per QALY gained), nonparametric bootstrapping was used to estimate variability around the arithmetic mean. ${ }^{30}$ Each of the 1000 bootstrap samples was adjusted for confounding variables (listed under Statistical Analysis section below). A net-benefit approach was used to measure the incremental costeffectiveness against a threshold value, $k$, which is often described as society's willingness to pay for one extra QALY gained. ${ }^{31}$ For a given threshold value $k$, an intervention would be considered to be cost effective if its net benefit is greater than $k^{32}$ The net benefit is defined by following relation:

$$
\begin{aligned}
\text { Net benefit } & =\left(k \times\left[\mathrm{QALYs}_{\mathrm{OCC}}-\mathrm{QALYs}_{\mathrm{non}-\mathrm{OCC}}\right]\right) \\
& -\left(\text { Total cost } \mathrm{OCC}_{\mathrm{OC}}-\text { Total }_{\text {cost }} \mathrm{non}-\mathrm{OCC}\right)
\end{aligned}
$$

Bootstrapped cost-effectiveness results were additionally used to generate cost-effectiveness acceptability curves, which show the probability of cost-effectiveness for different threshold values with regard to willingness to pay for one extra QALY. ${ }^{32}$ A threshold of $\$ 50,000$ per QALY is often used in international literature. ${ }^{33}$ If the ICER is below such a threshold value, the treatment can be regarded as cost effective. Given the evidence suggesting that $\$ 50,000$ per QALY is too low in the
United States, ${ }^{34}$ a value of $\$ 100,000$ was defined as the threshold for cost-effectiveness in the present analysis.

\section{Statistical analyses}

Baseline data were reported only descriptively as means and standard deviations (SDs) or as proportions. Study outcomes were first analyzed descriptively (e.g., number of affected patients, means and $95 \%$ confidence interval $[\mathrm{CI}]$ ). For the main analyses we tested the two-sided null hypothesis, $\mathrm{H}$ : mean total costs/effects in OCC group = mean total costs/effects in non-OCC group across the study period, and performed an analysis of covariance adjusted for age, sex, education, marital status, duration of CLBP treatment (in years) before study start, number of days with pain in the last 180 days, injections for back pain (yes/no), patients' expectation for improvement, smoking status, body-mass index (BMI), activity, and baseline value of the outcome (costs at baseline, utility at baseline). For inferential statistics, SPSS ${ }^{\odot}$ was used. Bootstrap analyses were conducted using MS $\mathrm{EXCEL}^{\odot}$ to model cost-effectiveness analyses.

\section{Results}

\section{Baseline characteristics}

The initial dataset included 309 patients in total. After exclusion of patients with missing QOL data at baseline $(n=19)$ and patients who had not provided QOL data at follow-up ( $n=12), 278$ patients (134 OCC, 144 non-OCC) were included in the present health economic analysis.

The baseline characteristics of study participants before study onset are summarized in Table 1. The OCC and nonOCC groups were comparable with regard to age, sex, and multiple back pain-related characteristics, including time since start of treatment, use of injections, and number of days with back pain in the past 180 days. However, patient baseline characteristics differed relevantly between groups in a number of other characteristics. Compared with the non-OCC group, OCC patients were more active, better educated, less likely to smoke, had lower BMI, were ethnically less diverse, less likely to be involved in litigation, and reported higher levels of marriage, employment, physical and mental QOL, expectations for improvement, and disease-related disability to work. Finally, differences in total costs between the patient groups before study onset were relevant with higher costs in the nonOCC group (OCC: mean \$3,723; non-OCC: mean \$2,933).

\section{Treatment utilization patterns}

As reported previously, ${ }^{17}$ the two groups were similar in their frequency of visits to specialists and physical therapists. However, the OCC group had a higher number of visits to primary care providers and integrative medicine providers during the course of the study. Regarding treatment received at the $\mathrm{OCC}$, the average number of visits to the OCC during the study was about 7.3 (SD: 6.7; range: 132). Most patients (about 69\%) had a treatment duration of 3 months or less and about $12 \%$ of patients had only a single visit to the OCC during the study timeframe.

\section{Cost outcomes}

During the first 3 months of the study, the total unadjusted direct mean costs per patient were higher for the OCC group 
Table 1. Baseline Characteristics of Participants in the Osher Clinical Center and Non-Osher Clinical Center Groups Included in Cost-Effectiveness Analysis

\begin{tabular}{|c|c|c|c|c|}
\hline \multirow[b]{2}{*}{ Sociodemographic variables } & \multicolumn{2}{|c|}{$O C C(\mathrm{n}=134)$} & \multicolumn{2}{|c|}{ Non-OCC $(\mathrm{n}=144)$} \\
\hline & & & & \\
\hline Male & 42 & 31.3 & 38 & 26.4 \\
\hline Female & 92 & 68.7 & 106 & 73.6 \\
\hline Current smoker, $n(\%)$ & 8 & 6.0 & 29 & 20.1 \\
\hline Ethnicity: Hispanic or Latino, $n(\%)$ & 8 & 5.6 & 22 & 15.3 \\
\hline Employed, $n(\%)$ & 81 & 60.4 & 51 & 35.4 \\
\hline Litigation, $n(\%)$ & 13 & 9.7 & 30 & 20.8 \\
\hline Marital status, $n(\%)$ & & & & \\
\hline Married or living with partner & 83 & 61.9 & 47 & 32.6 \\
\hline Education, $n(\%)$ & & & & \\
\hline High school level or below & 12 & 9.0 & 46 & 31.9 \\
\hline College or higher education & 122 & 91.0 & 98 & 68.1 \\
\hline Physically active, $n(\%)$ & & & & \\
\hline No & 26 & 19.4 & 44 & 30.6 \\
\hline Yes & 93 & 69.4 & 94 & 65.3 \\
\hline Injections ever, $n(\%)$ & & & & \\
\hline No & 72 & 53.7 & 78 & 54.2 \\
\hline Yes & 62 & 46.3 & 66 & 45.8 \\
\hline Age, mean (SD) & 50.18 & 16.65 & 52.08 & 15.88 \\
\hline BMI, mean (SD) & 26.02 & 5.48 & 30.29 & 7.97 \\
\hline CLBP treatment duration (in years) before study start, mean (SD) & 10.81 & 11.52 & 11.10 & 11.09 \\
\hline Expectation for improvement, mean (SD) & 6.02 & 2.63 & 2.92 & 3.10 \\
\hline Days with pain in last 180 days, mean (SD) & 144.08 & 43.67 & 153.52 & 46.06 \\
\hline Physical QOL component score, mean (SD) & 45.69 & 11.03 & 39.41 & 9.78 \\
\hline Mental QÖL component score, mean (SD) & 51.85 & 8.86 & 46.45 & 11.81 \\
\hline Utility at baseline, mean (SD) & 0.64 & 0.14 & 0.58 & 0.13 \\
\hline Number work incapacity days, mean (SD) & 6.13 & 18.51 & 2.45 & 11.94 \\
\hline Costs before study onset & & & & \\
\hline Daily activities, mean (SD) & $\$ 182.20$ & $\$ 627.20$ & $\$ 90.42$ & $\$ 398.10$ \\
\hline Devices, mean (SD) & $\$ 9.24$ & $\$ 28.94$ & $\$ 11.96$ & $\$ 29.95$ \\
\hline ER visits, mean $(\mathrm{SD})$ & $\$ 47.76$ & $\$ 186.17$ & $\$ 257.78$ & $\$ 728.22$ \\
\hline Hospitalizations, mean (SD) & $\$ 34.49$ & $\$ 399.30$ & $\$ 48.15$ & $\$ 331.24$ \\
\hline Injections, mean (SD) & $\$ 28.39$ & $\$ 63.69$ & $\$ 51.98$ & $\$ 219.88$ \\
\hline Medication, mean (SD) & $\$ 471.99$ & $\$ 2,021.58$ & $\$ 762.21$ & $\$ 2,386.25$ \\
\hline Office visits, mean (SD) & $\$ 813.84$ & $\$ 949.22$ & $\$ 779.64$ & $\$ 1,094.54$ \\
\hline Surgeries, mean (SD) & $\$ 0.00$ & $\$ 0.00$ & $\$ 18.43$ & $\$ 134.54$ \\
\hline Indirect costs, mean (SD) & $\$ 2,135.14$ & $\$ 6,166.35$ & $\$ 912.90$ & $\$ 3,950.74$ \\
\hline Total costs, mean (SD) & $\$ 3,723.05$ & $\$ 6,732.21$ & $\$ 2,933.46$ & $\$ 3,950.74$ \\
\hline
\end{tabular}

BMI, body-mass index; CLBP, chronic low back pain; ER, emergency room; OCC, Osher Clinical Center; QOL, quality of life; SD, standard deviation.

than the non-OCC group (difference per patient $\$ 947[95 \%$ CI: 500-2,449]). For the period 3-6 months the cost difference was reduced to $\$ 235$ (95\% CI: -282 to 752 ), and for 6-12 months there was a direct cost savings of $\$ 391(95 \%$ CI: $-1,078$ to 1,861$)$ observed. For unadjusted indirect costs due to reduced work productivity, such a switch was not observable and these costs were higher in the OCC group over the complete study duration.

As seen in Table 2, the annual unadjusted total costs over the complete study period were higher for patients in the OCC group compared with the non-OCC group (OCC: mean $\$ 9,106$ [95\% CI: 6,233-11,980], non-OCC: mean $\$ 6,283$ [95\% CI: 4,522-8,043]). Adjusting costs for relevant differences in baseline characteristics between groups resulted in statistically significant higher total costs in the OCC group compared with the non-OCC group over the complete study period (OCC: adjusted mean $\$ 11,527$ [95\% CI: 7,76615,287]; non-OCC: $\$ 6,811$ [95\% CI: 3,763-9,858]; $p=0.024)$. When looking into specific cost items, adjusted costs were not statistically different for medical devices, ER visits, injections, medications, and indirect costs, but statistically higher in the OCC group for daily activities, hospitalizations, office visits, and surgeries.

\section{Effectiveness outcomes}

Over 12 months, patients in the OCC group experienced more unadjusted QALYs compared with the non-OCC group (OCC: mean 0.6989 [95\% CI: 0.6776-0.7202], nonOCC: mean 0.6205 [95\% CI: 0.5975-0.6435]). After adjusting for baseline differences, the QALYs during the study were not statistically different between the groups (OCC: 
Table 2. Unadjusted and Adjusted Annual Costs and Resource Consumption per Patient

\begin{tabular}{|c|c|c|c|c|}
\hline \multirow[b]{2}{*}{ Cost item } & \multirow[b]{3}{*}{$\mathrm{N}^{\mathrm{a}}$} & \multirow{3}{*}{$\frac{O C C}{\operatorname{Mean}(95 \% C I)}$} & \multicolumn{2}{|r|}{ Non-OCC } \\
\hline & & & & Mean $(95 \%$ CI) \\
\hline $\mathrm{p}$-Value for adjusted mean ${ }^{\mathrm{b}}$ & & & $\mathrm{N}^{\mathrm{a}}$ & Adjusted mean (95\% CI) \\
\hline $\begin{array}{l}\text { Daily activities } \\
p=0.003\end{array}$ & 60 & $\begin{array}{l}\$ 486.63(294.16 \text { to } 679.10) \\
\$ 419.42(181.26 \text { to } 657.58)\end{array}$ & 25 & $\begin{array}{l}\$ 77.08(32.02 \text { to } 122.15) \\
\$ 20.09(-174.14 \text { to } 214.31)\end{array}$ \\
\hline $\begin{array}{l}\text { Devices } \\
p=0.216\end{array}$ & 60 & $\begin{array}{l}\$ 65.73(7.80 \text { to } 123.67) \\
\$ 100.44(33.45 \text { to } 167.44)\end{array}$ & 53 & $\begin{array}{l}\$ 42.53(-1.28 \text { to } 86.35) \\
\$ 54.55(0.08 \text { to } 109.01)\end{array}$ \\
\hline $\begin{array}{l}\text { ER visits } \\
p=0.428\end{array}$ & 7 & $\begin{array}{c}\$ 47.76(10.73 \text { to } 84.80) \\
\$ 418.12(245.84 \text { to } 590.41)\end{array}$ & 33 & $\begin{array}{l}\$ 433.33(271.08 \text { to } 595.59) \\
\$ 494.06(352.44 \text { to } 635.68)\end{array}$ \\
\hline $\begin{array}{l}\text { Hospitalizations } \\
p=0.033\end{array}$ & 12 & $\begin{array}{l}\$ 1,234.89(-151.09 \text { to } 2,620.87) \\
\$ 2,108.07(432.47 \text { to } 3,783.68)\end{array}$ & 7 & $\begin{array}{l}\$ 224.69(48.47 \text { to } 400.91) \\
\$ 119.89(-1,236.95 \text { to } 1,476.72)\end{array}$ \\
\hline $\begin{array}{l}\text { Injections } \\
p=0.270\end{array}$ & 36 & $\begin{array}{l}\$ 101.95(49.61 \text { to } 154.29) \\
\$ 177.88(31.52 \text { to } 324.25)\end{array}$ & 36 & $\begin{array}{l}\$ 249.25(116.44 \text { to } 382.07) \\
\$ 267.16(148.17 \text { to } 386.14)\end{array}$ \\
\hline $\begin{array}{l}\text { Medications } \\
p=0.634\end{array}$ & 124 & $\begin{array}{r}\$ 970.31(578.89 \text { to } 1,361.73) \\
\$ 2,189.38(387.07 \text { to } 3,979.69)\end{array}$ & 134 & $\begin{array}{l}\$ 2,553.99(1,160.64 \text { to } 3,947.33) \\
\$ 2,656.21(1,199.04 \text { to } 4,113.38)\end{array}$ \\
\hline $\begin{array}{l}\text { Office visits } \\
p<0.001\end{array}$ & 127 & $\begin{array}{l}\$ 2,430.21(1,954.45 \text { to } 2,905.96) \\
\$ 2,904.28(2,294.86 \text { to } 3,516.69)\end{array}$ & 114 & $\begin{array}{l}\$ 1,271.39 \text { (988.00 to } 1,554.78) \\
\$ 1,279.30 \text { (784.56 to } 1,774.04)\end{array}$ \\
\hline $\begin{array}{l}\text { Surgeries } \\
p=0.006\end{array}$ & 12 & $\begin{array}{l}\$ 165.76(73.43 \text { to } 258.10) \\
\$ 246.38(134.61 \text { to } 358.14)\end{array}$ & 5 & $\begin{array}{l}\$ 27.89(-4.31 \text { to } 60.08) \\
\$ 74.51(-15.89 \text { to } 164.91)\end{array}$ \\
\hline $\begin{array}{l}\text { Indirect costs } \\
p=0.433\end{array}$ & 42 & $\begin{array}{l}\$ 3,603.09(1,520.40 \text { to } 5,685.78) \\
\$ 2,851.41(338.54 \text { to } 5,364.27)\end{array}$ & 25 & $\begin{array}{l}\$ 1,402.46(551.35 \text { to } 2,253.57) \\
\$ 1,761.72(-262.16 \text { to } 3,785.61)\end{array}$ \\
\hline $\begin{array}{l}\text { Total costs } \\
p=0.024\end{array}$ & 134 & $\begin{array}{r}\$ 9,106.33(6,232.64 \text { to } 11,980.02) \\
\$ 11,526.73(7,766.14 \text { to } 15,287.31)\end{array}$ & 144 & $\begin{array}{l}\$ 6,282.62(4,521.82 \text { to } 8,043.42) \\
\$ 6,810.63(3,763.15 \text { to } 9,858.11)\end{array}$ \\
\hline $\begin{array}{l}\text { Annual resource consumption } \\
\text { Number ER visits } \\
p=0.428\end{array}$ & 7 & $\begin{array}{l}0.07(0.02 \text { to } 0.13) \\
0.65(0.38 \text { to } 0.92)\end{array}$ & 33 & $\begin{array}{l}0.68(0.42 \text { to } 0.93) \\
0.77(0.55 \text { to } 0.99)\end{array}$ \\
\hline $\begin{array}{l}\text { No. of hospitalizations } \\
p=0.007\end{array}$ & 12 & $\begin{array}{l}0.13(0.05 \text { to } 0.20) \\
0.25(0.15 \text { to } 0.35)\end{array}$ & 7 & $\begin{array}{l}0.05(0.01 \text { to } 0.08) \\
0.10(0.01 \text { to } 0.18)\end{array}$ \\
\hline $\begin{array}{l}\text { Days of hospitalization } \\
p=0.033\end{array}$ & 12 & $\begin{array}{l}0.53(-0.05 \text { to } 1.13) \\
0.91(0.19 \text { to } 1.64)\end{array}$ & 7 & $\begin{array}{l}0.10(0.02 \text { to } 0.17) \\
0.05(-0.54 \text { to } 0.64)\end{array}$ \\
\hline $\begin{array}{l}\text { No. of different drugs } \\
p=0.350\end{array}$ & 124 & $\begin{array}{l}4.77(4.15 \text { to } 5.39) \\
6.11(5.21 \text { to } 7.01)\end{array}$ & 134 & $\begin{array}{l}5.57(4.94 \text { to } 6.20) \\
5.65(4.92 \text { to } 6.38)\end{array}$ \\
\hline $\begin{array}{l}\text { No. of office visits } \\
p \leq 0.001\end{array}$ & 127 & $\begin{array}{l}22.80(18.35 \text { to } 27.26) \\
26.87(21.11 \text { to } 32.62)\end{array}$ & 114 & $\begin{array}{l}10.97(8.31 \text { to } 13.64) \\
11.22(6.54 \text { to } 15.90)\end{array}$ \\
\hline $\begin{array}{l}\text { Number work incapacity days } \\
p=0.615\end{array}$ & 30 & $\begin{array}{l}7.95(2.22 \text { to } 13.67) \\
7.77(0.52 \text { to } 15.01)\end{array}$ & 21 & $\begin{array}{l}3.34(0.39 \text { to } 6.29) \\
5.75(-0.09 \text { to } 11.60)\end{array}$ \\
\hline
\end{tabular}

${ }^{\text {a}}$ Patients actually being affected by the item, mean values are referring to the total $n$ by group.

bAdjusted for: age, sex, education, marital status, time since LBP treatment start, number of days with pain in the last 180 days, injections for back pain (yes/no), patients' expectation for improvement, smoking status, BMI, activity, baseline value of the outcome.

95\% CI, 95\% confidence interval; BMI, body-mass index; ER, emergency room; LBP, low back pain; OCC, Osher Clinical Center.

adjusted mean 0.6420 [95\% CI: 0.6204-0.6635]; non-OCC: adjusted mean 0.6312 [95\% CI: $0.6135-0.6489] ; p=0.362$ ).

There was a greater increase in back function in the OCC group compared with the non-OCC group (adjusted differences in RDQ scale OCC: -1.726 [95\% CI: -2.973 to -0.480$]$ and non-OCC +0.548 points [95\% CI: -0.506 to 1.603 ]; resulting in a clinically meaningful adjusted group difference of 2.275 points [95\% CI: 3.630 to $0.919 ; p=0.001])$. The proportion of RDQ responders ( $\geq 30 \%$ improvement) was $20.3 \%$ in the OCC group and $10.6 \%$ in the non-OCC group $(p=0.011)$. Results were similar for the BOP scale (OCC: -1.536 points [95\% CI: -2.220 to -0.852 ] and non-OCC: -0.414 points [95\% CI: -0.969 to 0.141$]$; adjusted group difference of 1.122 points [95\% CI: 1.865 to $0.379 ; p=0.003]$ ). The proportion of BOP responders was $39.8 \%$ in the OCC group and $20.9 \%$ in nonOCC group $(p=0.001)$.

\section{Cost-effectiveness}

As shown above, the modestly greater improvement in QALYs in the OCC treatment compared with the non-OCC treatment (adjusted models) was associated with higher costs. The primary ICER was $\$ 436,676$ per QALY gained in the OCC group. A cost-effectiveness plane of this estimate and its uncertainty, based on bootstrapping (1000 estimates), is shown in Figure 1.

Nearly all bootstrap samples are located in the upper quadrants, indicating that cost savings associated with the intervention are unlikely. Three quarters $(76.3 \%)$ of the 


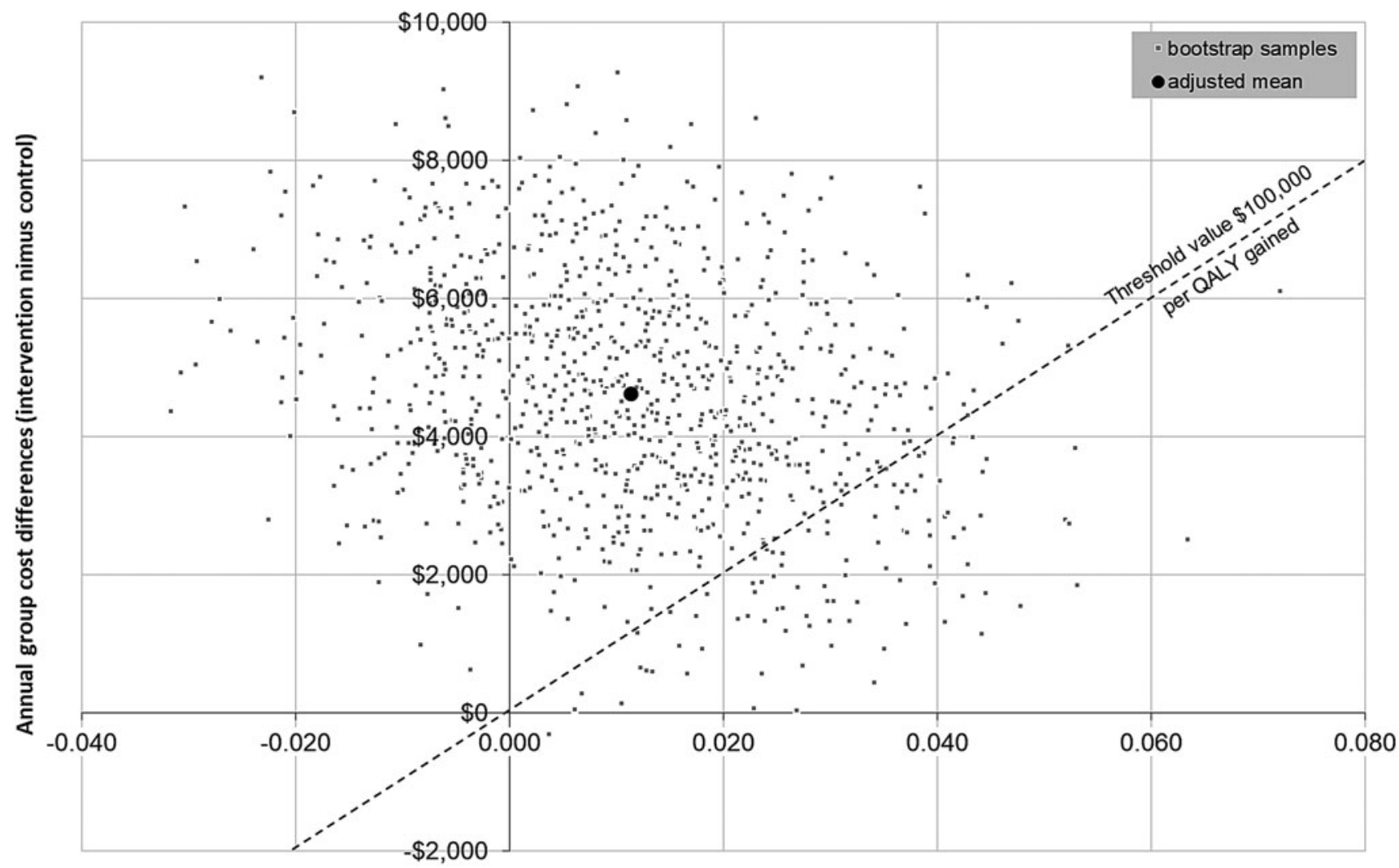

Annual group QALY differences (intervention minus control)

FIG. 1. Cost-effectiveness plane. Cost-effectiveness of OCC versus non-OCC treatment of chronic low-back pain based on bootstrapping (1000 estimates). OCC, Osher Clinical Center; QALYs, quality-adjusted life years.

bootstrapped QALY differences are located in the upper right quadrant indicating a better effectiveness at higher costs in the OCC group. The remaining bootstrap samples show lower QALYs compared with the non-OCC group. When using the net-benefit approach to measure the cost-effectiveness probability against a range of threshold values for willingness-topay for one extra QALY gained (Fig. 2) the cost-effectiveness probability reaches $9.8 \%$ assuming the a priori-defined willingness-to-pay of $\$ 100,000$ per QALY gained.

Exploratory analyses for ICERs based on adjusted RDQ group differences (in favor of OCC) showed that the cost for a one-point reduction on the RDQ was $\$ 2,073$ and the cost per additional RDQ responder was $\$ 48,620$. Parallel exploratory ICERs for adjusted BOP scores (in favor of OCC) indicated the cost for a one-point reduction in BOP was $\$ 4,203$, and the cost per additional BOP responder was $\$ 24.953$.

\section{Discussion}

This observational study characterized back pain-related costs and cost-effectiveness for care delivered at a multimodal integrative care clinic based in a tertiary care hospital (OCC), as compared with care delivered in the same hospital but outside the OCC. Over a 12-month period, analyses adjusted for important baseline differences indicate statistically significant and greater CLBP-related total costs per patient in the OCC group compared with the non-OCC group. In contrast, QALYs were only marginally improved in the OCC group. Consequently, the ICER estimate of cost per QALY gained (our primary cost-effectiveness outcome) was high $(\$ 436,676)$ and not below the a priori-defined threshold for cost-effectiveness. However, the two primary back pain-specific clinical outcomes evaluated, RDQ and BOP (coprimary outcomes in our clinical study), ${ }^{17}$ showed clinically meaningful and statistically greater improvements at 12 months in the OCC versus the non-OCC group. ICERs based on these outcomes (exploratory analyses) provide alternative cost-effectiveness estimates.

Between-study comparisons of cost-effectiveness are difficult due to variability in populations studied (e.g., acute vs. CLBP), therapeutic exposures (types and doses), costs considered (e.g., general medical vs. back pain specific), and time frames of observation. Nevertheless, our finding of lack of cost-effectiveness based on QALYs for the model of integrated care delivered at the OCC contrasts with other studies, which have reported that adding individual or multiple integrative care therapies (e.g., spinal manipulation, acupuncture, yoga, cognitive behavioral therapy, mindfulness-based stress reduction) to usual routine care can be cost effective. ${ }^{35-40}$ For example, the UK BEAM trial reported that a combination of spinal manipulation and exercise had a greater impact on QALYs and was cost effective compared with usual care. ${ }^{36}$ However, this study targeted both subacute and CLBP patients and included general health care costs in addition to back pain-specific costs.

More recently, the ATEAM study also reported that the use of certain combinations of integrative therapies (Alexander technique) with exercise were clinically more effective than usual care, with ICERs for the best therapy combination 


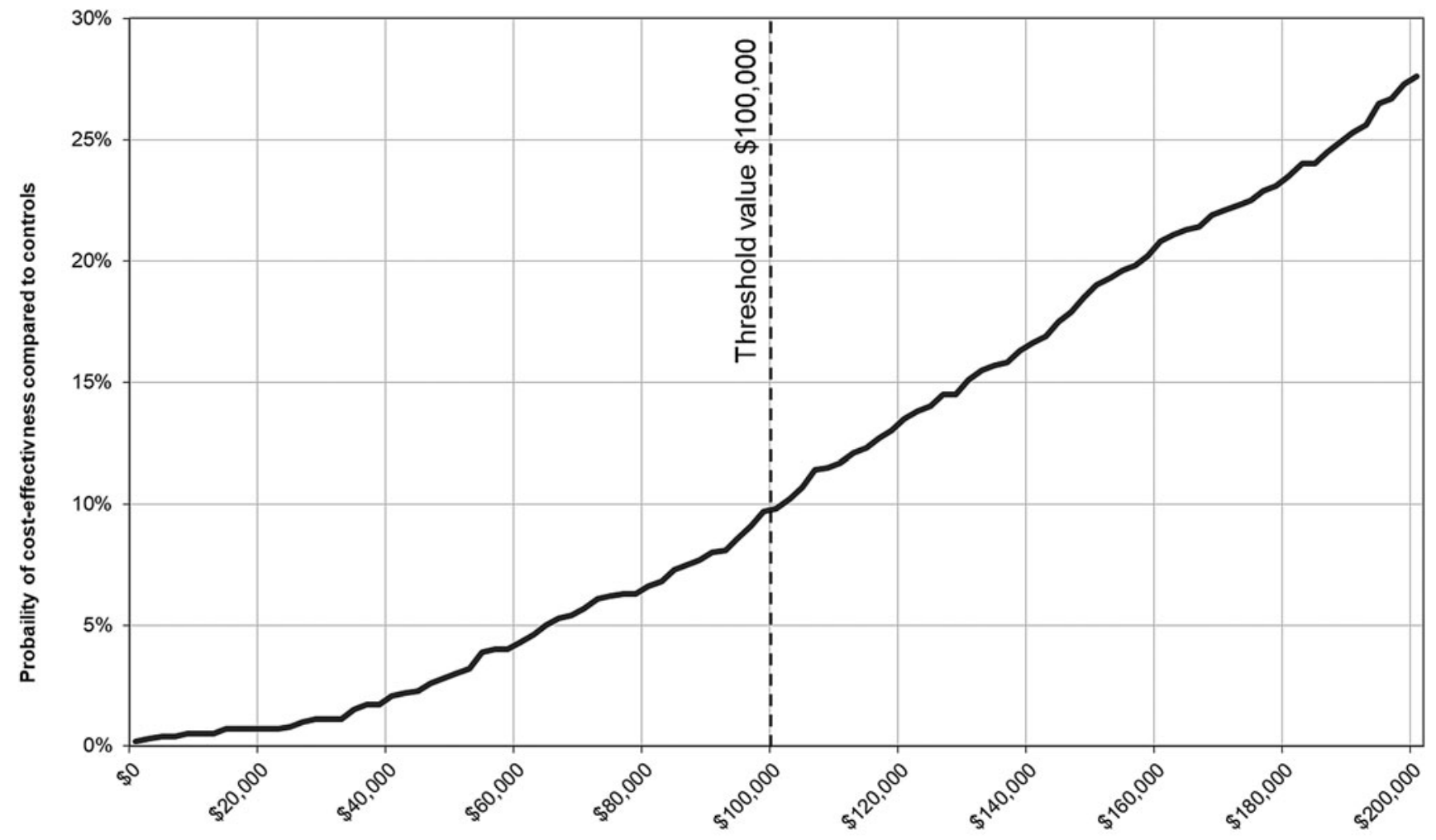

Willingness to pay for one additional QALY (threshold value)

FIG. 2. Probability of cost-effectiveness against different willingness-to-pay threshold values. QALYs, quality-adjusted life years.

estimated to be $£ 5,332$ per QALY gained. ${ }^{37}$ Of note, QALYs gained in this study were as high as 0.065 , which contrasts with the smaller between-group difference in QALY of 0.011 observed in our study. Paralleling our findings, Kominski et al. $^{41}$ reported that the addition of chiropractic care, with or without ancillary physical modalities, was associated with higher medical costs and was not cost-effective.

A closer look at both costs and clinical outcomes, and especially their changes over 12 months, reveals a noteworthy trend. When averaged over the 12 -month period, CLBPrelated costs were $\$ 4,716$ higher in the OCC versus the nonOCC group. The largest contributors to between-group total cost differences, in order of magnitude and all with higher costs in OCC were, office visits $(\$ 1,625)$, indirect costs $(\$ 1,090)$, hospital visits (non-ER) (\$871), daily activities (\$394), and surgeries (\$172). However, when only looking at direct costs, the magnitude of between-group difference in total expenditures decreased over time, from $\$ 947$ to $\$ 235$ per patient at 3 and 6 months, respectively, and then showed a mean cost savings of $\$ 391$ in the OCC group at 12 months.

A closer look at patterns of medical expenditures over time reveals that the observed savings in direct costs in the OCC during later observational periods were largely driven by reduced usage of CLBP-related medications (data not shown). The number of OCC patients that reported taking CLBP medications at 3, 6, and 12 months was 126, 105, and 90, respectively. In contrast, the number of non-OCC patients that reported taking CLBP medications did not decline as dramatically between 3, 6, and 12 months, and was 138, 120,119, respectively. This observation warrants further investigation in future trials.
In addition to potentially impacting cost savings, the observed reduced dependence on pain medications in the OCC suggests that integrative models of CLBP care may contribute to the reduction of overreliance on potentially dangerous and addictive medications, known to have significant implications to both individuals and society. ${ }^{42-44}$

Magnitudes of between-group differences in QALYs were small and did not differ markedly over the 12 months. In contrast, the magnitude of between-group differences in back pain-related disability in favor of OCC was more marked. Adjusted between-group differences in RDQ were 0.30, 0.95, and 2.08 at 3-, 6-, and 12-month assessments, respectively. ${ }^{17}$

It is noteworthy that most patients in the OCC group were treated at the OCC only during the first 3 months of the study, suggesting a potential delayed impact on disability. One interpretation of these findings is that sustained and longer-term improvements in RDQ in the OCC group contributed to the reductions in medical spending observed only during the 6- to 12-month observation period. These longer-term clinical effects may be attributable to the explicit emphasis on self-care that was a primary focus of the team-based training at the OCC. ${ }^{15,16}$ Future studies of the OCC should include an extended (e.g., 2-year) observation period to better evaluate potential longer-term impacts on effectiveness and cost-effectiveness.

The noteworthy trend in improvement in RDQ over time, and its association with decreased medical spending also led us to explore alternative metrics of cost-effectiveness based on disability, as has been done in prior back pain studies. ${ }^{37,45,46}$ ICERs based on adjusted RDQ group differences showed that cost for a one-point reduction in the RDQ was $\$ 2,073$ and cost per 
additional RDQ responder was $\$ 48,620$. Because there is little consensus on cost-effectiveness thresholds for utility values other than QALYs, these findings are difficult to interpret.

However, for comparison, Hollinghurst et al. estimated that incremental costs (including both direct and indirect) for a point reduction in RDQ were $£ 448, £ 113$, and $£ 61$ for massage therapy, Alexander lessons, and exercise, when these were compared with usual care in patients with CLBP. ${ }^{37}$ Other studies, including ICERs based on alternative metrics of disability or pain scales suggest that estimates of cost-effectiveness based on measures other than QALY can provide valuable and complementary data for assessing the clinical and economic evidence base for alternative LBP treatment strategies. ${ }^{4,46}$

Our study has a number of limitations. While our analyses controlled for important known baseline differences between the OCC- and non-OCC-treated groups, it is possible that other residual confounders, unknown or unmeasured, could have contributed to our observed group differences in this observational study. We were unable to obtain information about referral patterns to the OCC and other non-OCC clinics; therefore, we cannot rule out the possibility of selection bias.

Baseline differences in sociodemographic factors, and in particular, with wealthier and more educated patients choosing care at the OCC, could have influenced patterns of medical spending that were not fully accounted for in our modeling. With these limitations in mind, however, our findings suggest modest improvements in functional status and BOP, and trends toward savings in direct cost during latter periods of observation, supporting the potential value of evaluating multimodal integrative models for the treatment of CLBP in more definitive randomized controlled trials.

In addition, this study focused on the effectiveness of care delivered by a multimodal integrative care clinic in a single academic center hospital. Large-scale multisite randomized trials would be needed to make broader generalizations regarding the effectiveness and cost-effectiveness of multimodal integrative approaches to CLBP. Toward this end, two complementary approaches could be used.

First, and more pragmatic, would be to replicate at a larger scale the strategy employed in this study. CLBP patients at multiple academic medical centers would be randomly assigned to receive care at an integrative medicine clinic or through conventional care within the same institution. The inclusion of multiple integrative medical clinics, each with their unique model of delivery, would afford good generalization regarding the benefits of integrative medicine approaches for CLBP.

Alternatively, and less pragmatic, would be to evaluate the impact of specific subsets of combinations of therapies most commonly used at integrative medicine centers. In the case of the OCC, this would be chiropractic plus acupuncture, or chiropractic plus mind-body training. ${ }^{17}$ Findings from this approach would result in more focused recommendations regarding the use of multimodal approaches in the treatment of CLBP, and also lay a better foundation for exploring mechanisms underlying observed synergistic clinical and economic benefits.

\section{Conclusions}

Patients who utilize integrative care in an academic hospital setting differ substantially from those who utilize conventional treatments. When baseline sociodemographic and back pain-related differences are accounted for, selfreported costs were higher in the integrative care group with no apparent effect on QALYs, despite meaningful improvements in CLBP-related disability and BOP at 12 months. Future studies evaluating potential benefits of integrative care models for the management of CLBP should employ randomized clinical designs, longer observational periods, and explore multiple metrics of cost-effectiveness.

\section{Acknowledgment}

This work was supported by grants R01-AT005065 and K24-AT009282 from the National Center for Complementary and Integrative Health at the National Institutes of Health.

\section{Author Disclosure Statement}

No competing financial interests exist.

\section{References}

1. Speed C. Low back pain. BMJ 2004;328:1119-1121.

2. Shmagel A, Foley R, Ibrahim H. Epidemiology of chronic low back pain in US adults: Data from the 2009-2010 National Health and Nutrition Examination Survey. Arthritis Care Res 2016;68:1688-1694.

3. Hoy D, March L, Brooks P, et al. The global burden of low back pain: Estimates from the Global Burden of Disease 2010 study. Ann Rheum Dis 2014;73:968-974.

4. Morlion B. Chronic low back pain: Pharmacological, interventional and surgical strategies. Nat Rev Neurol 2013; 9:462-473.

5. Deyo RA, Von Korff M, Duhrkoop D. Opioids for low back pain. BMJ 2015;350:g6380.

6. Murthy V, Sibbritt DW, Adams J. An integrative review of complementary and alternative medicine use for back pain: A focus on prevalence, reasons for use, influential factors, self-perceived effectiveness, and communication. Spine J 2015;15:1870-1883.

7. Clarke TC, Nahin RL, Barnes PM, Stussman BJ. Use of complementary health approaches for musculoskeletal pain disorders among adults: United States, 2012. Natl Health Stat Report 2016:1-12.

8. Eisenberg DM, Davis RB, Ettner SL, et al. Trends in alternative medicine use in the United States, 1990-1997: Results of a follow-up national survey. JAMA 1998;280:1569-1575.

9. Wolsko PM, Eisenberg DM, Davis RB, et al. Patterns and perceptions of care for treatment of back and neck pain: Results of a national survey. Spine 2003;28:292-297; discussion 298.

10. Wolsko PM, Eisenberg DM, Davis RB, et al. Insurance coverage, medical conditions, and visits to alternative medicine providers: Results of a national survey. Arch Intern Med 2002;162:281-287.

11. Ghildayal N, Johnson PJ, Evans RL, Kreitzer MJ. Complementary and alternative medicine use in the US adult low back pain population. Glob Adv Health Med 2016;5:69-78.

12. Walker BF, French SD, Grant W, Green S. Combined chiropractic interventions for low-back pain. Cochrane Database Syst Rev 2010;4:CD005427.

13. Rubinstein SM, van Middelkoop M, Assendelft WJ, et al. Spinal manipulative therapy for chronic low-back pain. Cochrane Database Syst Rev 2011;2:CD008112.

14. Herman PM, Poindexter BL, Witt CM, Eisenberg DM. Are complementary therapies and integrative care cost-effective? 
A systematic review of economic evaluations. BMJ Open 2012;2:e001046.

15. Eisenberg DM, Kaptchuk TJ, Post DE, et al. Establishing an integrative medicine program within an academic health center: Essential considerations. Acad Med 2016; 91:1223-1230.

16. O'Connor BL, Eisenberg DM. Case study: Osher Clinical Center for Complementary and Integrative Medical Therapies (Chapter 5). In: Beyond the Checklist: What Else Healthcare Can Learn from Aviation Safety and Teamwork. Ithaca, NY: Cornell University Press, 2013.

17. Wayne PM, Eisenberg DM, Osypiuk K, et al. A multidisciplinary integrative medicine team in the treatment of chronic low-back pain: An observational comparative effectiveness study. J Altern Complement Med 2018;24: 781-791.

18. Ware J, Kosinski M, Keller SD. A 12-item short-form health survey: Construction of scale and preliminary tests of reliability and validity. Med Care 1996;34:220-223.

19. Deyo RA, Dworkin SF, Amtmann D, et al. Report of the NIH Task Force on research standards for chronic low back pain. J Pain 2014;15:569-585.

20. Blue Cross Blue Shield of Massachusetts. Typical costs for common medical services. Online document at: www .bluecrossma.com/blue-iq/pdfs/TypicalCosts_89717_042709 .pdf, accessed June 25, 2015.

21. Weiss AE, Elixhauser A. Overview of hospital stays in the United States, 2012. HCUP Statistical Brief No. 180, 2014 Online document at: www.hcup-us.ahrq.gov/reports/ statbriefs/sb180-Hospitalizations-United-States-2012.pdf, accessed June 25, 2015.

22. $\mathrm{CPT}^{\circledR} /$ Medicare payment search. Online document at: https://apps.ama-assn.org/CptSearch/user/search/cptSearch .do, accessed July 22, 2015.

23. IBM Micromedex ${ }^{\circledR}$ RED BOOK Online ${ }^{\circledR}$. Online document at: www.micromedexsolutions.com, accessed April 20, 2016.

24. Hawkins K, Wang S, Rupnow MF. Indirect cost burden of migraine in the United States. J Occup Environ Med 2007; 49:368-374.

25. Brazier JE, Roberts J. The estimation of a preferencebased measure of health from the SF-12. Med Care 2004; 42:851-859.

26. Richardson G, Manca A. Calculation of quality adjusted life years in the published literature: A review of methodology and transparency. Health Econ 2004;13:1203-1210.

27. Patrick D, Deyo RQ, Atlas SJ, et al. Assessing healthrelated quality of life in patients with sciatica. Spine 1995; 20:1899-1909.

28. Cherkin DC, Sherman KJ, Balderson BH, et al. Effect of mindfulness-based stress reduction vs cognitive behavioral therapy or usual care on back pain and functional limitations in adults with chronic low back pain: A randomized clinical trial. JAMA 2016;315:1240-1249.

29. Sanders GD, Neumann PJ, Basu A, et al. Recommendations for conduct, methodological practices, and reporting of costeffectiveness analyses: Second panel on cost-effectiveness in health and medicine. JAMA 2016;316:1093-1103.

30. Efron B. Bootstrap methods: Another look at the jackknife. Ann Stat 1979;7:1-26.

31. Zethraeus N, Johannesson M, Jonsson B, et al. Advantages of using the net-benefit approach for analysing uncertainty in economic evaluation studies. Pharmacoeconomics 2003; 21:39-48.
32. Lothgren M, Zethraeus N. Definition, interpretation and calculation of cost-effectiveness acceptability curves. Health Econ 2000;9:623-630.

33. Greenberg D, Winkelmayer W, Neumann PJ. Prevailing judgments about society's willingness to pay for a QALY or life-year gained. Ital J Public Health 2005;2(Suppl 1):301.

34. Neumann PJ, Cohen JT, Weinstein MC. Updating costeffectiveness-The curious resilience of the $\$ 50,000$-perQALY threshold. N Engl J Med 2014;371:796-797.

35. Miyamoto GC, Lin CC, Cabral CMN, et al. Costeffectiveness of exercise therapy in the treatment of nonspecific neck pain and low back pain: A systematic review with meta-analysis. Br J Sports Med 2018;53:172-181.

36. UK BEAM Trial Team. United Kingdom back pain exercise and manipulation (UK BEAM) randomised trial: Cost effectiveness of physical treatments for back pain in primary care. BMJ 2004;329:1381.

37. Hollinghurst S, Sharp D, Ballard K, et al. Randomised controlled trial of Alexander technique lessons, exercise, and massage (ATEAM) for chronic and recurrent back pain: Economic evaluation. BMJ 2008;337:a2656.

38. Witt CM, Jena S, Selim D, et al. Pragmatic randomized trial evaluating the clinical and economic effectiveness of acupuncture for chronic low back pain. Am J Epidemiol 2006; 164:487-496.

39. Herman PM, Anderson ML, Sherman KJ, et al. Costeffectiveness of mindfulness-based stress reduction versus cognitive behavioral therapy or usual care among adults with chronic low back pain. Spine 2017;42:1511-1520.

40. Thomas KJ, MacPherson H, Ratcliffe J, et al. Longer term clinical and economic benefits of offering acupuncture care to patients with chronic low back pain. Health Technol Assess 2005;9:iii-iv, ix-x, 1-109.

41. Kominski GF, Heslin KC, Morgenstern H, et al. Economic evaluation of four treatments for low-back pain: Results from a randomized controlled trial. Med Care 2005;43:428-435.

42. Atluri S, Sudarshan G, Manchikanti L. Assessment of the trends in medical use and misuse of opioid analgesics from 2004 to 2011. Pain Physician 2014;17:E119-E128.

43. Lipman A, Webster L. The economic impact of opioid use in the management of chronic nonmalignant pain. J Manag Care Spec Pharm 2015;21:891-899.

44. Brady KT, McCauley JL, Back SE. Prescription opioid misuse, abuse, and treatment in the United States: An update. Am J Psychiatry 2016;173:18-26.

45. Niemisto L, Rissanen P, Sarna S, et al. Cost-effectiveness of combined manipulation, stabilizing exercises, and physician consultation compared to physician consultation alone for chronic low back pain: A prospective randomized trial with 2-year follow-up. Spine 2005;30:1109-1115.

46. Whitehurst DG, Lewis M, Yao GL, et al. A brief pain management program compared with physical therapy for low back pain: Results from an economic analysis alongside a randomized clinical trial. Arthritis Rheum 2007;57:466-473.

Address correspondence to: Peter M. Wayne, PhD Osher Center for Integrative Medicine Brigham and Women's Hospital and Harvard Medical School 900 Commonwealth Avenue, 3rd Floor Boston, MA 02215

E-mail: pwayne@bwh.havard.edu 